\section{Herztod beim Sex ist wohl eher eine Rarität}

\author{
Auch wenn gerade Männer sich diesbezüglich manchmal Sorgen machen \\ mögen: Die Wahrscheinlichkeit, beim Sex einen tödlichen Herzstillstand zu \\ erleiden, ist äußerst gering.
}

$\mathrm{D}$ e mit sexueller Aktivität verbundene Aufregung kann auch das Herz ziemlich unter Stress setzen. Gleichwohl herrscht in der Medizin weitgehend Konsens darüber, dass es selbst Patienten mit Herzinfarkt, die sich nach dem Ereignis wieder erholen, nicht verwehrt sein sollte, auch weiterhin sexuell aktiv zu sein. Denn die Wahrscheinlichkeit, dass die sexuelle Lust dem Herzen so zusetzt, dass es zum Herzstillstand kommt, ist sehr gering.

Wenn es aber in seltenen Fällen zu einem solchen Ereignis kommt, dann ha- ben Männer klar die schlechteren Karten. Das zeigen Ergebnisse der Studie Oregon SUDS (Sudden Unexpected Death Study).

Forscher des Cedars-Sinai Medical Center in Los Angeles um Dr. Sumeet Chugh haben 4.557 Fälle von plötzlichem Herzstillstand analysiert. Darunter waren lediglich 34 Herzstillstände (0,7\%), die in zeitlichem Zusammenhang mit sexueller Aktivität aufgetreten. Das absolute Risiko war demnach extrem niedrig. Von den 34 Herzstillständen waren 18 während der sexuellen Aktivität und 15 in den ersten Minuten nach dem Sex auf- getreten; in einem Fall war die zeitliche Zuordnung nicht genau zu bestimmen.

In 32 der 34 Fälle (94\%) waren Männer betroffen. Von allen 34 Betroffenen hatten $29 \%$ eine KHK und $26 \%$ eine symptomatische Herzinsuffizienz in ihrer Vorgeschichte, die Mehrzahl nahm kardiovaskulär wirksame Medikamente ein.

Die Forscher verweisen auf einen weiteren Aspekt ihrer Studie. Darin zeigte sich nämlich, dass Wiederbelebungsmaßnahmen nur in einem Drittel aller Fälle vorgenommen worden waren selbst dann, wenn andere Personen anwesend waren. Sie erinnern daran, dass es wichtig sei, in den Bemühungen fortzufahren, die Öffentlichkeit über die Wichtigkeit der kardiopulmonalen Reanimation bei plötzlichem Herzstillstand aufzuklären, egal unter welchen Umständen er auftrete. Peter Overbeck

\title{
Schützt Botox vor postoperativem Vorhofflimmern?
}

Botulinumtoxin hat auch in der Kardiologie erste Erfolge gefeiert. In Pilotstudien war das Neurotoxin in der Lage, das Auftreten von Vorhofflimmern nach Herz-Operationen zu unterdrücken. Eine aktuelle randomisierte Studie bringt nun Ernüchterung.

n Botulinumtoxin, besser bekannt als Botox, steckt viel mehr als nur ein Faltenglätter. Mittlerweile haben auch Kardiologen das Gift für ihre Zwecke ins Visier genommen. Erprobt wird das Neurotoxin als Therapieoption im Rahmen von Herzoperationen mit der Hoffnung, damit das Auftreten von postoperativem Vorhofflimmern unterdrücken zu können. Erste Ergebnisse aus kleineren Pilotstudien waren durchaus vielversprechend.

Doch wie so häufig in der klinischen Forschung stellt sich das Konzept nun in einer größeren randomisierten Studie namens TNTPAOF als nur wenig wirksam heraus. Die Studie wurde auf dem AHA vorgestellt.

\section{Postoperatives Vorhofflimmern ist ein klinisches Problem}

Als Ursache für ein postoperatives Vorhofflimmern wird ein Ungleichgewicht des sympathischen und parasympathischen Nervensystems vermutet. Durch seine anticholinerge Wirkung könnte Botulinumtoxin der Arrhythmie-Entwicklung entgegenwirken.

In der doppelblinden randomisierten TNT-POAF-Studie gelang dies allerdings nur bedingt. 130 Probanden bekamen während einer KoronararterienBypass-Operation oder Klappen-Operation entweder Onabotulinum Toxin A in das epikardiale Fettgewebe injiziert oder eine Salzlösung.

\section{Ergebnis statistisch nicht signifikant}

Eine Woche nach dem Eingriff hatten $36,5 \%$ der Patienten mit der Botox-Behandlung Vorhofflimmern entwickelt, in der Kontrollgruppe waren es 47,8\%. Der
Unterschied von $11 \%$ war statistisch nicht signifikant $(p=0,19)$. Signifikant geringer war die Dauer der ersten Vorhofflimmern-Episode (1,9 vs. 5,5 Stunden, $\mathrm{p}=0,01$ ).

Trotz dieses unbefriedigenden Studienergebnisses gibt der Vortragende Prof. Nathan Waldron das Konzept nicht auf. Die Studie sei womöglich underpowered gewesen, um eine geringe, aber klinisch bedeutsame Risikoreduktion belegen zu können. Darüber hinaus handelte es sich um eine Hochrisikopopulation, bei der es im Vorfeld bereits zu Vorhofflimmern-Episoden gekommen war.

Seiner Ansicht nach besteht noch Hoffnung, dass epikardial injiziertes Botulinumtoxin das Risiko für postoperatives Vorhofflimmern senken kann. Diese Annahme müsse man nun in weiteren, großangelegten Studien prüfen.

Die gute Nachricht: In dieser Studie waren keine Sicherheitsrisiken durch die Botox-Behandlung erkennbar. Weder die Krankenhausverweildauer noch die postoperativen Komplikationsraten und die Mortalität unterschieden sich zwischen beiden Gruppen.

Ob die Sicherheit aber auch langfristig gewährleistet ist, bleibt abzuwarten.

Veronika Schlimpert 